\title{
Atypical adolescent sexual behaviour: A study of involvement in triolism and same gender sex in Lagos, Nigeria
}

\author{
Kunnuji, Michael \\ Department of Sociology, University of Lagos, Akoka - Yaba, Nigeria. E-mail: mkunnuji@unilag.edu.ng, \\ nuunayt@yahoo.com, michaelkunnuji@gmail.com. Tel: +234 - 08023540699.
}

Accepted 16 December, 2011

\begin{abstract}
A very wide range of sexual practices are available to adolescents. The options range from the socially approved act of absolute sexual chastity to uncommon ones such as group sex and same-gender sex. Many studies in the area of sexuality in Nigeria proceed from the premise that adolescent sexual behaviour hardly ever goes beyond premarital heterosexual intercourse whether it is voluntary, forced or transactional. Others are aware that other possibilities exist but hardly seek to document them. Thus, very little is known about the extent to which adolescents are involved in these "unusual" or atypical sexual practices. Using data from a survey of 614 sexually active adolescents in Lagos metropolis, this article fills the lacuna in the literature on the prevalence of atypical sexual practices in an urban setting in Nigeria. The results show that about $14 \%$ of the subjects had been involved in at least one of the two atypical sexual acts and about $1 \%$ had been involved in both acts. The study concludes therefore that sexual practices labelled "uncommon", "atypical" or "unusual" may not be as uncommon as people often imagine.
\end{abstract}

Key words: Unusual sexual behaviour, uncommon sexual behaviour, adolescent sexuality, group sex, homosexuality.

\section{INTRODUCTION AND BACKGROUND OF THE STUDY}

Sexual behaviour is a major subset of human behaviour. Reproduction and the continuity of the human race are tied primarily to this aspect of behaviour as most conceptions occur as a result of sexual intercourse. Conceptions occurring independent of sexual intercourse are few and unaffordable to a large proportion of people, particularly in the global South where poverty levels are high. When this is considered, it becomes clear that sexual activities play a major role in perpetuating the human race. Apart from the interest in sexual activities for the purpose of procreation, pleasure also attracts humankind to sexual activities. On the other hand, the spread of HIV/AIDS which occurs largely through sexual activities (although there are other non-sexual means of transmission) suggests that sexual behaviour is a twoedged sword which is equally capable of decimating human populations. Countries with very high proportions of people living with HIV/AIDS in Africa have also experienced regression in demographic transition such that an era of very high birth and death rates which had once been overcome reoccurred in their histories. Such countries would have been recording negative growth rates but for interventions targeted at sexual behavioural change. The two-edged nature of human sexuality is therefore one reason for the attention it has received among researchers and advocacy groups in all parts of the world. Studies are often targeted at young people because of their large share of the total population in many countries of the world and their peculiar reproductive health needs. In Nigeria for instance, young people within the age bracket of 10 to 24 years account for about $34 \%$ of the country's total population (PRB, 2006). Several studies have emphasised the reproductive health needs of this group.

The Demographic and Health Survey of 2003 points out that the median ages at onset of sex for men and women generally are 20.4 and 17.3 years respectively while the median ages at entry into marriage are 26.4 and 18.5 
years for men and women [National Population Commission (NPC) (Nigeria) and ORC Macro, 2004). The gap between the age at onset of sex and entry into marriage often makes adolescents vulnerable to sexually transmitted infections as reproductive health services are within some social contexts made available only to people in marital unions and some young people avoid approaching reproductive health centres for fear of being labelled immoral (Erulkar et al., 2005). For this and other reasons, use of condom at first sex is put at just $6.5 \%$ for girls within the age bracket of 15 to 19 years and $10.6 \%$ for boys. These studies further show differentials in sexual behaviour across wealth quintiles, levels of education, residence and age categories. The Nigerian Federal Ministry of Health made similar findings through a survey in 2006 showing further how vulnerable the group is. About 41 and $84 \%$ of young females within the age brackets of 15 to 19 and 20 to 24 years had had sex. Among boys within the 15 to 19 and 20 to 24 years age brackets, 20 and $63 \%$ had had sex. Among sexually experienced girls within the 15 to 19 and 20 to 24 age groups, 35 and $73 \%$ had had premarital sex in the last 12 months preceding the study while among boys, $15 \%$ of those within the 15 to 19 age group had had sex in the last 12 months and $53 \%$ of young men within the age bracket of 20 to 24 years had had sex in the last 12 months. The study shows further that among sexually experienced youths (15 to 19 years), $8 \%$ had contracted STIs in the last 12 months preceding the survey. For the 20 to 24 age group, 1 in 10 had contracted an STI (Federal Ministry of Health, 2006). While researchers agree that adolescent sexual behaviour needs to be studied, it is very difficult to agree on definitions of what constitute a given sexual act like "having sex", being "sexually experienced" and/or "sexually active" (Kelly, 2004: 5). Many researchers therefore simply ask questions about experience of penetrative sexual intercourse. Respondents may however not perceive same gender sexual intimacy as "having sex". The consequence is that subjects may not volunteer such information since they are not asked. Persons who have had same gender sexual experience only may thus be left out of the sexually active population and their sexual health needs are often not documented through research. Another sexual practice that often escapes the eyes of researchers in the area of adolescent sexual behaviour is involvement in sexual encounters involving more than two persons, usually two or more persons of the same gender and one of the opposite gender. The number can also be varied for as long as there are willing partakers. It is against this background that this study seeks to fill the knowledge gaps on these two atypical or "uncommon" sexual practices among young people in Lagos, Nigeria.

Clift et al. (2009) found that atypical sexual interests as manifested in arousal to sexual stimuli depicting children or coercivelsex is a predictor of sexual offending and recidivism. Through an appraisal of several studies on sexual offending, Seto and Lalumiere (2010) agree that atypical sexual interest as a variable has effect on sexual offending. These contributions call attention to the ugly side of atypical sexual interests and practices. Put differently, atypical sexual interests may suggest the presence of a high tendency to be involved in sex crime. This gives justification to the interest in atypical sexual behaviour since treatment of sexual offending and recidivism may benefit from proper documentation of the prevalence of atypical sexual behaviour. This study does not seek to test the assertion that atypical sexual interest and sexual offending are correlates; however, rather, it seeks to document the prevalence of atypical sexual behaviour in the study population. In some countries of the world, reproductive health programmes have been designed to meet the needs of persons involved in the aforementioned sexual practices which have the potential of increasing vulnerability to 'sexually transmitted infections'. The United States, for instance, has a Centre for Disease Control and Prevention (CDC) which has a programme - the CDC National Prevention Information Network. This programme offers reference and referral services for information on HIV/AIDS and other diseases. The National Prevention Information Network has a campaign component which addresses particular gaps in health communication literature on gay men who have sex with men, their knowledge of STDs and their communication preferences and it protects the health of gay, bisexual and other men who have sex with men (www.cdcpin.org/scripts/campaign/strategy.asp, 2011; www.cdc.gov/msmhealth/msm-programs.htm, 2011).

There are no similar programmes in Nigeria and the laws of the land proscribe same gender sexual activities.

\section{Theoretical underpinnings}

In seeking explanations to the different sexual behavioral options available to young people, Kirkpatrick's exposé shall be explored briefly. For youth, Kirkpatrick (1955) identifies eight (8) sex conduct choices which are available: i) premarital chastity; ii) early marriage; iii) premarital heterosexual monogamous sex affairs; iv) casual heterosexual premarital sex affairs; v) relations with prostitutes; vi) petting to climax; vii) homoeroticism (homosexual affair); viii) and autoeroticism (masturbation). He further classifies these options into three. Options 1 and 2 are classified as conformity to sex codes; options 3, 4, 5 and 6 are classified under rebellious premarital heterosexual relationships; while options 7 and 8 are categorized as homoerotic and autoerotic outlets of the sex urge. Each option has its pros and cons as shown by Kirkpatrick (1955) and they are not to be perceived as exclusive sexual conduct choices. He further makes nine assertions concerning sex codes. These assertions are: 1) every culture has mores concerning sex; 2 ) the sex mores vary widely from 
group to group; 3) a sex code reflects human and social needs and likewise local imperatives as interpreted in some particular social situation; 4) emerging codes may have functional utility for a particular situation, or they may express mere superstition and magical thinking; 5) the sex mores as part of the control culture may lag with respect to the utilitarian (technological) culture...; 6) codes are rarely adjusted to individual differences, for exceptions weaken the sanction of codes; 7) the individual is psychologically bound by the mores of his group regardless of their merits, and therefore he rebels with effort, over protest and a guilty conscience; 8) conformity to the sex mores reduces confusion, uncertainty and spares the effort of working out a personal code; 9) halfway adjustments are often especially disturbing, with the advantages of neither rebellion nor conformity... (Kirkpatrick, 1955: 329).

Having discussed these options and the implications of the social contexts within which they are created and in which they operate, the repression theory is examined. The repression theory flows from the argument that antisocial sexual behavior results from social inhibitions. In other words, atypical sexual behaviors are less likely to occur in situations where there is minimal repression on sexuality. To fully appreciate this explanation, the ideas of Ollman (1978) are useful. He asserts that all members of society (children inclusive) desire sexual intercourse, and in adolescence, long before marriage is possible, this desire becomes overpowering. The sexual life of young people, he concludes, is characterized by extreme frustration and guilt, because sexual activity of one kind or another occurs despite the social prohibition (Ollman, 1978). Same gender encounters, which again are widespread in adolescence... are even more laden with guilt feelings." Such practices are thus done secretly, in haste and worry over being discovered (Ollman, 1978: 187). From the repression perspective, it is argued that sexual appetite is never in excess, and is never furious, save when it has been excessively repressed. The frenzied hunger we see sexual urge exhibiting in the form of different antisocial behaviour marks the hideous repression to which society subjects it. This is not a normal state of the appetite. Rather, these atypical expressions of sexuality result from the unnatural repression imposed upon it by the exigencies of society (Marcuse, 1966; Ollman, 1978). Marcuse (1966: 202) stresses this further in stating that it is the suppression of sexuality that results in "the sadistic and masochistic orgies of desperate masses, of "society elites," of starved bands of mercenaries, of prison and concentration camp guards." This way, the theory shows that atypical sexual behavior is an active expression of resistance to repression. It explains why adolescents appear sexually recalcitrant and the observed sexual recalcitrance further calls for repression from society.

The result is a cumulative circular interaction between repression and sexual deviance. Is the repression hypothesis applicable in the explanation of atypical sexual behaviour among urban adolescents in Nigeria? This question shall be addressed in this study.

\section{METHODS}

Data for this study come from a larger survey of 1120 adolescents within the age bracket of 10 to 24 years in the city of Lagos, Nigeria. For the purpose of this analysis, only 614 subjects who said they had had sex and responded appropriately (that is not those who failed to respond) to questions on involvement in samegender sex and sex involving more than two persons are included. The respondents were selected through a multi-stage sampling exercise in which five local government areas (LGAs) were randomly selected out of 16 metropolitan LGAs. From each selected LGA, streets were listed and sampled randomly while households were systematically selected before eligible respondents were randomly drawn. Informed consent was sought and obtained from adolescents 18 years and above while parental consent was obtained for adolescents below the age of 18 years before interviews were conducted. All interviews were conducted outside hearing distance, away from third parties and only successful interviews (that is interviews in which reliable information were obtained on the core concerns of the study) were processed for analysis using the Statistical Package for Social Sciences, Version 10.

A standardized interview schedule containing questions on respondents' background information and sexual activities from the time of first sexual encounters to the time of the study was employed in eliciting information from the subjects.

\section{Inclusion and exclusion criteria}

The following criteria were employed for inclusion in the study:

i) Age as at last birthday falls within the bracket of 10 to 24 years; ii) Respondent had been sexually involved (with someone of the same/different sex);

iii) Respondent resides in Lagos metropolis;

iv) Respondent agrees to be part of the study and provide answers to questions central to the study.

\begin{abstract}
Analysis
The simple frequency and percentage analysis is used in the discussion of the prevalence of atypical sexual practices. The chi square test is used to establish association between involvement in atypical sexual behaviour and independent variables such as age, gender and age at onset of sex. Also, involvement in at least one atypical sexual practice was measured at the nominal level with involvement coded "1" and its absence "0". This makes it suitable for logistic regression analysis, with age at onset of sex and gender as independent variables.
\end{abstract}

\section{RESULTS AND DISCUSSION}

A total of 357 male and 257 female adolescents were found to be sexually active in a sample of 1120 . Three respondents abstained from the question on involvement in same-gender sex while four abstained from the question on involvement in group sex. In all, 608 
respondents answered both questions and were included in the analysis on atypical sexual behaviour which measures involvement in at least one of the two sexual practices. Among the 608 subjects, $86 \%$ had never been involved in any of the two practices, $10 \%$ had been involved in group sex only, $4 \%$ had been involved in same-gender sex only while five (about $1 \%$ had been involved in both group sex and same-gender sex. On the whole, $14 \%$ of the respondents had been involved in at least one of the two practices. There is a greater reason to accept as true that subjects who abstained from the questions on involvement in same-gender sex and group sex had been involved in those practices than to accept as true that they had never been involved in them. The Nigerian society in general does not approve of the practices as socially acceptable behaviours and persons not involved in them are not likely to have any inhibition to saying they had never been involved in them.

To affirm this, about $91 \%$ of adolescents in Lagos metropolis argue that homosexuality is improper. In other words, the figures made available by this study may be considered as the lower estimate of involvement in atypical sexual practices in the study population.

\section{Prevalence of and factors associated with group sex}

As stated earlier, one in every ten of the respondents reported involvement in sex involving more than two people at the same time. Using the chi-square test, gender was found to be significantly associated with involvement in group sex $\left(x^{2}=21.643 ; p<0.001\right)$. While $14 \%$ of male adolescents had been involved in group sex, just $3 \%$ of girls had been involved. The study shows further that young people who had become sexually active by the age of 16 years reported greater involvement in group sex $\left(x^{2}=7.932 ; p<0.05\right)$. About $15 \%$ of the subjects who had initiated sexual activities by the age of 16 years had been involved in group sex while just about $8 \%$ of those who initiated sex after the age of 16 years reported involvement in group sex. Contrary to the conjecture that people who attend single sex educational institutions are more exposed to atypical sexual behaviour and likely to exhibit such, the study shows that there is no significant relationship between attendance of single sex educational institution and involvement in group sex.

\section{Prevalence of and factors associated with same gender sex}

About $5 \%$ of the subjects had been involved in same gender sex. In order to shed light on some predictors of involvement in atypical sexual behaviour, conjectures were made. Gender was considered a factor to explore due to the differentials in societal expectations in terms of sexual behaviour (Dixon-Mueller, 1996). Gender is not significantly associated with same-gender sex. Developmental stage is also not significantly associated with same gender sex. The study shows further that there is no significant relationship between developmental stage and atypical sexual practices. Similarly, timing of onset of sex and attendance of has no association with same-gender single sex educational institution were found to have no significant relationship with involvement in same gender sex. This suggests that factors associated with involvement in same gender sex require further studies.

\section{DISCUSSION OF FINDINGS}

Although developmental stage (that is early, mid or late adolescence) was conjectured to be associated with atypical sexuality, statistical tests show that there is no significant relationship between developmental stage and atypical sexual practices. The relationship between timing of onset of sexual activity and atypical sexual practices was also explored. Adolescents who had become sexually active by the age of 16 years reported greater involvement in group sex, but not same gender sex. The study also shows that there is no significant relationship between attendance of same sex educational institution and involvement in atypical sexual behaviour. The logistic regression test further affirms that gender and age at onset of sex are predictors of involvement in at least one atypical sexual behaviour. Male adolescents were found to be more than twice (2.405 times) as likely to have been involved in one atypical sexual behaviour than female adolescents while adolescents who had initiated sexual activity by the age of 16 years were found to be almost twice (1.675 times) more likely to have taken part in one atypical sexual behaviour than those who initiated sexual activity later. The findings thus negate the repression theory in a sense as female adolescents known to be under more severe sexual repression in the Nigerian society were not found to have exhibited greater atypical sexual behaviour than males who have a higher level of freedom to experiment in the area of sexuality.

In addition, adolescents who had become sexually active and/or expressive cannot be said to have experienced greater repression than those who remained chaste until the age of 17 years. Yet, those who had been sexually active since age 16 exhibited greater atypical sexual behaviour. This study thus promotes a counterrepression thesis. Sexual behaviour is a very broad subset of human behaviour. It is obviously not limited to penetrative heterosexual intercourse and it is almost impossible to create a list of all sexual acts. Yet, sexual activities considered uncommon are not less important and attempts to study human sexuality and reproductive health behaviour ought to factor in atypical sexual behaviours. The reproductive health needs of persons 
involved in atypical sexual activities should also be factored into interventions to ensure a society where the welfare of all, including minority groups and persons with special sexual/reproductive health needs are met. It was for this reason that this study sought to explore the prevalence of two atypical sexual activities. The study has shown that although these activities are far from being consensual in the Nigerian society, they are not as uncommon as one might imagine.

\section{Limitations}

The study relies on self reports and thus stands the risk of taking as true underreported non-consensual sexual activities. It also suffers from inaccuracy resulting from ability to recall age at onset of sex; although it is difficult for adolescents to forget whether they had been involved in group sex or same-gender sexual activities.

\section{Conclusion}

This study set out to explore adolescents' involvement in same-gender sex and group sex as indicators of involvement in atypical sexual activities among young people in Lagos. It considered as sexually active all respondents who had been involved in heterosexual as well as same-gender sex. The study reports an atypical sexual activity prevalence rate of $14 \%$, with males who had initiated sexual activities by the age of 16 years being the most likely group of adolescents to take part in atypical sexual activities. The findings of the study question the applicability of the repression theory within the study population. The repression explanation suggests, within the context of this study, that atypical sexual practices are manifestations of strong inhibitions on sexual behaviour. A corollary to this is the notion that sexual practices that do not enjoy societal approval are bound to be prevalent within social contexts where sexuality is most restrained. By the same token, sexual liberalism reduces sexual deviance. This study shows that there is no empirical basis for this position within the context of adolescent sexuality in metropolitan Lagos.

\section{REFERENCES}

Clift RJW, Rajlic G, Gretton HM (2009). Discriminative and Predictive Validity of the Penile Plethysmograph in adolescent sex offenders. Sex. Abuse: J. Res. Treat., 21(3): 335-362.

Dixon-Mueller R (1996). The Sexuality Connection in Reproductive Health. In Zeidenstein S \& Moore K (eds). Learning about Sexuality: A Practical Beginning. New York: The Population Council, International Women's Health Coalition. pp. 137-157

Erulkar AS, Onoka CJ, Phiri A (2005). What is Youth Friendly? Adolescents' Preferences for Reproductive Health Services in Kenya and Zimbabwe. Afr. J. Rep. Health, 9(3): $51-58$.

Federal Ministry of Health [Nigeria]: (2006). National HIV/AIDS \& Reproductive Health Survey, (NARHS) 2005. Abuja: Federal Ministry of Health.

Kelly GF (2004). Sexuality Today: The Human Perspective. Boston: McGraw Hill. p.5

Kirkpatrick C (1955). The Family as Process and Institution. New York: The Ronald Press Company. pp. 213.-215

Marcuse H (1966). Eros and Civilization. Boston: Beacon Press. p.202

National Population Commission (NPC) [Nigeria] \& ORC Macro (2004). Nigeria Demographic and Health Survey 2003. Calverton, Maryland: National Population Commission and ORC Macro.

Ollman B (1978). Social and Sexual Revolution: Essays on Marx and Reich. Montreal: Black Rose Press: 187

Population Reference Bureau (2006). World's Youth 2006 Data Sheet. Washington DC: PRB.

Seto MC, Lalumiere ML (2010). What is so special about male adolescent sexual offending? A review and test of explanations through meta-analysis. Psych. Bull., 136(4): 526-575. 